\section{D) Check for updates}

Cite this: Polym. Chem., 2022, 13 100

Received 10th September 2021, Accepted 19th November 2021 DOI: 10.1039/d1py01227d rsc.li/polymers

\title{
Triggered and monitored drug release from bifunctional hybrid nanocomposites $\uparrow$
}

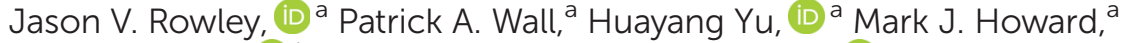 \\ Daniel L. Baker, (D) b Alexander Kulak, ${ }^{a}$ David C. Green (D) *a and \\ Paul D. Thornton (iD *a
}

\begin{abstract}
Polymer-coated carbon dot-containing calcium carbonate nanoparticles are reported as unique nanocomposites capable of encapsulating a chemotherapeutic drug and displaying afterglow behaviour. The poly(amino acid) polymeric component enhances nanoparticle dispersion and drug retention in aqueous solution, and can be designed to be cleavable to enable doxorubicin release under acidic conditions. The encapsulated carbon nanodots offer fluorescence and brief afterglow to the nanocomposites at neutral $\mathrm{pH}$, which is lost when the nanocomposites encounter acidic $(\mathrm{pH} 5)$ solution. The loss of drug molecules, and fluorescence and phosphorescence provided by the carbon dots, in acidic solution ensures that the reported materials have potential promise as early-stage candidates as devices for monitored payload delivery to acidic environments.
\end{abstract}

\section{Introduction}

Polymeric materials may be designed to release molecular cargo upon interaction with a range of stimuli including changes in environmental $\mathrm{pH}^{1-3}$ and temperature, ${ }^{4-6}$ or the presence of (bio)molecules including enzymes ${ }^{7}$ and reducing agents. $^{8,9}$ The controlled and prolonged release of preserved therapeutic, fragrant, and biocidal agents from a polymeric core is exploited in medicinal, household care, and personal care products. Poly(amino acids) are particularly well-suited for use as such polymeric carriers owing to their tailorable functionality and excellent biocompatibility. ${ }^{10}$ Poly(amino acid) synthesis by the ring-opening polymerisation (ROP) of amino acid $N$-carboxyanhydrides (NCAs) ensures that polymers of narrow dispersity are produced at high yield with the amino acid chirality retained, ${ }^{11,12}$ whilst polymer degradation results in the reformation of non-toxic amino acids in the absence of toxic side-products. ${ }^{13,14}$ Innovative poly(amino acid)-based materials include stimuli-responsive nanoparticles for controlled payload release, hydrogels proposed as scaffolds for tissue regeneration, and non-cytotoxic organogels. ${ }^{15-17}$

Polymer-coated inorganic nanoparticles are a class of nanocomposites that have the potential to be used for the encapsulation, and subsequent controlled release, of guest molecules. For

${ }^{a}$ School of Chemistry, University of Leeds, Leeds, LS2 9JT, UK.

E-mail: p.d.thornton@leeds.ac.uk, david.green@hh.se

${ }^{b}$ School of Physics and Astronomy, University of Leeds, Leeds, LS2 9JT, UK

$\dagger$ Electronic supplementary information (ESI) available. See DOI: 10.1039/ d1py01227d instance, poly(amino acid)-coated silica particles have been demonstrated to encapsulate and selectively release the model compound rhodamine b. ${ }^{18}$ Therapeutic-loaded nanocomposites have great potential as drug delivery vehicles whereby the polymeric shell enhances nanoparticle biodistribution, biocompatibility, and prevents premature drug metabolism. ${ }^{19}$ A stimuliresponsive polymeric shell may act as an actuator for controlling drug release on-command, in response to a target stimulus. ${ }^{20}$ Such controlled release enables reduced drug dosage, which consequently mitigates side-effects caused by the interactions between non-target cells and the therapeutic molecule. ${ }^{21}$ Polymer-coated inorganic nanoparticles that possess a stimuliresponsive, degradable, and non-cytotoxic polymeric shell are therefore highly desirable as potential drug delivery vehicles.

Whilst drug delivery may be a primary function of such nanocomposites but the ability to track and trace their distribution and function in vivo is also desirable. Fluorescent dyes and nanoparticles are currently used, as they report immediately and can be detected precisely using inexpensive and commonly available microscopic and spectroscopic methods. Quantum dots are common, however they frequently rely on heavy metals for these properties which renders them cytotoxic, and unsuitable for use in vivo without encapsulation or modification. ${ }^{22}$ On the contrary, fluorescent carbon nanodots (CNDs) express biocompatibility, low toxicity, chemical stability, photoluminescence, and ease of synthesis. ${ }^{23}$ Consequently, CNDs are used for a wide-range of applications including bioimaging, ${ }^{24}$ drug delivery, ${ }^{25}$ light-emitting devices $^{26}$ and as photocatalysts. ${ }^{27,28}$ When certain CNDs are composited with a polymer such as poly(vinyl alcohol ${ }^{29}$ or 
polyurethane,$^{30}$ or an inorganic host material, ${ }^{31}$ they display bimodal fluorescence and persistent afterglow which is visible after the removal of the stimulation source. In bioimaging, the ability to image with the excitation laser switched off would help to eliminate noise due to short-lifetime autofluorescence common to biomolecules and live cells, ${ }^{32,33}$ and thus in theory provides access to more sensitive imaging and detection methods. ${ }^{34}$ CNDs are therefore well suited for reporting the degradation of their afterglow-activating host since their release into the external environment eliminates their afterglow behaviour.

Calcium carbonate $\left(\mathrm{CaCO}_{3}\right)$ is an attractive inorganic core material thanks to its biocompatibility and ability to incorporate guest particles, including CNDs. ${ }^{35-37}$ Recently, persistent afterglow from CNDs was activated when they were incorporated into $\mathrm{CaCO}_{3}$ nanoparticles (CaNPs), where the dense internal environment of the inorganic crystal promoted longlifetime phosphorescence emission. However bare CaNPs typically aggregate and recrystallise in aqueous solution and therefore require stabilisation. Surface functionalisation of CaNPs with fatty acids, ${ }^{38}$ an organotitanate coupling agent ${ }^{39}$ or organosilanes ${ }^{40}$ have been shown to increase the compatibility and stabilise CaNP dispersions in non-aqueous media. In contrast, there are only a few examples of enhanced stability of CaNP suspensions in aqueous media. Dong et al. developed a stable poly(ethylene glycol) (PEG)-modified CaNP nanocomposite loaded with doxorubicin (Dox) and a photosensitiser, ${ }^{35}$ where payload release was triggered by reduced solution $\mathrm{pH}$ and in situ monitoring was enabled by magnetic resonance imaging. Although the material shows great promise as a theranostic agent, PEG is associated with the production of antiPEG antibodies and accelerated blood clearance. ${ }^{41}$ Therefore, alternative, degradable polymers that enable CaNP dispersion in aqueous solution are sought.

Herein, we describe the grafting of poly(amino acids) to CaNPs that are enriched with CNDs and exhibit both fluorescence and afterglow. The resulting nanocomposite comprises a polymer shell which aids their dispersion in water, and provides the mechanism through which drug molecule entrapment and release is achieved. Poly(amino acid) coatings were created by both direct polymer growth from the CaNP surface and grafting pre-formed poly(amino acids) to CaNPs through noncovalent interactions. The latter enabled straightforward polymer characterisation, and in both cases polymers that contained ester linking groups were grafted to CaNPs, rendering the polymer shell susceptible to acid-catalysed hydrolysis. Finally, the potential application of the nanocomposites as theranostic devices was initially assessed by observing the $\mathrm{pH}$-controlled retention and release of the chemotherapeutic drug Dox.

\section{Experimental section}

\subsection{Synthesis of $\mathrm{CND} / \mathrm{CaNPs}$}

$\mathrm{CND} / \mathrm{CaNPs}$ were synthesised via the carbonation method as reported previously. ${ }^{31}$ Briefly, a $5 \mathrm{mg} \mathrm{mL}{ }^{-1}$ CND stock solution was prepared by suspending $0.1 \mathrm{~g}$ folic acid in $20 \mathrm{~mL}$ deionised (DI) water and adding $\mathrm{NaOH}$ until dissolution occurred. The resulting solution was then heated to $200{ }^{\circ} \mathrm{C}$ in a hydrothermal bomb for $12 \mathrm{~h}$ to yield a stock solution of folic acidderived CNDs. A UV-Vis spectrum was obtained showing two plateaus at 258 and $323 \mathrm{~nm}$ (Fig. S18†). FTIR $\nu_{\max }$ (solid) of lyophilised CNDs: $3250 \mathrm{~cm}^{-1}$ (bm, OH and $\left.\mathrm{NH}\right), 1659 \mathrm{~cm}^{-1}$ (m, $\mathrm{C}=\mathrm{O}$ ) and $1579 \mathrm{~cm}^{-1}(\mathrm{~s}, \mathrm{~N}-\mathrm{H})$. Separately, $\mathrm{CaCO}_{3}$ was decomposed to $\mathrm{CaO}$ in a furnace at $900{ }^{\circ} \mathrm{C}$ over $16 \mathrm{~h} .0 .44 \mathrm{~g} \mathrm{CaO}$ was then added to $50 \mathrm{ml}$ degassed and distilled DI water under vigorous stirring over $16 \mathrm{~h}$ at room temperature to produce a $\mathrm{Ca}$ $(\mathrm{OH})_{2}$ slurry containing approx. $157 \mathrm{mM} \mathrm{Ca}^{2+} .2 \mathrm{~mL}$ of CND stock solution was added in one go while the slurry was vigorously stirred. Immediately after, a mixture of $\mathrm{N}_{2}$ and $\mathrm{CO}_{2}(3: 1$ $\mathrm{v} / \mathrm{v}$ ) was then bubbled through the slurry at a rate of $1 \mathrm{~L} \mathrm{~min}^{-1}$ under stirring. The gas flow was continued until the solution $\mathrm{pH}$ reached 7, immediately after which the CND/CaNPs were collected and washed by centrifugation $(1 \times 10 \mathrm{~min}, 8000 \mathrm{rpm}$ from water, and $1 \times$ from ethanol), and dried.

\subsection{Surface-functionalisation of $\mathrm{CND} / \mathrm{CaNPs}$ with $\mathrm{ABPA}$ and HUPA}

CND/CaNPs (5 mg) and 4-aminobutylphosphonic acid (ABPA) $(5 \mathrm{mg}, 0.03 \mathrm{mmol})$ or 11-hydroxyundecylphosphonic acid (HUPA) $(5 \mathrm{mg}, 0.20 \mathrm{mmol})$ were weighed into a glass vial and suspended in DI water $(5 \mathrm{~mL})$ with the aid of an ultrasonic bath. The ABPA- or HUPA-functionalised CND/CaNPs were collected by centrifuge, washed with DI water and ethanol, and dried in an oven at $50^{\circ} \mathrm{C}$.

\subsection{Synthesis of nanocomposites CND/CaNP-ABPA-PPhe $e_{4}-b$ - PSar $_{16}$ and CND/CaNP-ABPA-PBLG ${ }_{18}$-b-PSar ${ }_{16}$ : the grafting- from approach}

As a representative example, ABPA-functionalised CND/CaNPs ( $0.26 \mathrm{~g} \mathrm{CND/CaNPs}$ and $0.06 \mathrm{~g}, 0.4 \mathrm{mmol}, 1$ eq. ABPA) were suspended in anhydrous dimethylformamide (DMF, $20 \mathrm{~mL}$ ) and added to an oven-dried, $\mathrm{N}_{2}$-flushed Schlenk tube. The NCA (L-phenylalanine (Phe)-NCA: $0.37 \mathrm{~g}, 2.0 \mathrm{mmol}, 5$ eq. or $\gamma$-benzyl-L-glutamate (BLG)-NCA: $1.77 \mathrm{~g}, 6.7 \mathrm{mmol}, 23 \mathrm{eq}$.) was dissolved in anhydrous DMF $(20 \mathrm{~mL})$ and also added to the Schlenk tube. The polymerisation was stirred at room temperature with a flow of $\mathrm{N}_{2}$ until FTIR spectroscopy determined that all NCA had reacted due to the absence of peaks corresponding to the anhydride groups of the NCA monomer. Sarcosine (Sar)-NCA was then dissolved in anhydrous DMF $(10 \mathrm{~mL})$, added to the Schlenk tube and stirred at room temperature with a flow of $\mathrm{N}_{2}$. Once again, FTIR spectroscopy was used to determine that all NCA monomer had reacted, the reaction mixture was reduced to dryness, and the resulting solid washed three times in chloroform (centrifugation for 30 minutes each time at $4500 \mathrm{rpm}$ ). The nanocomposites CND/ CaNP-ABPA-poly(Phe $)_{4}-b$-poly(Sar $)_{16} \quad$ (CND/CANP-ABPA-PPhe $4^{-}$ $b$-PSar ${ }_{16}$ ) and CND/CaNP-ABPA-poly(BLG) ${ }_{18}-b-$ PSar $_{16}$ (CND/ CaNP-ABPA-PBLG $18_{18}-b$-PSar ${ }_{16}$ ) were collected by centrifugation and dried in a vacuum oven at $45^{\circ} \mathrm{C}$. 


\subsection{Synthesis of nanocomposite CND/CaNP-HUPA-PSar ${ }_{82}$ :} the grafting-from approach

The NCA ROP initiated from a hydroxyl group was carried out as reported previously, with modifications. ${ }^{42,43}$ HUPA-functionalised CND/CaNPs (0.02 g $\mathrm{CND} / \mathrm{CaNPs}$ and $0.02 \mathrm{~g}$, $0.08 \mathrm{mmol}, 1$ eq. HUPA) were suspended in anhydrous DMF (25 $\mathrm{mL}$ ) in a Schlenk tube. The milky-white coloured solution was stirred under $\mathrm{N}_{2}$ before Sar-NCA $(0.44 \mathrm{~g}, 3.81 \mathrm{mmol}, 48$ eq.) was added. Methanesulfonic acid in anhydrous DMF solution $\left(0.02 \mathrm{mg} \mathrm{mL}^{-1}, 1 \mathrm{~mL}\right)$ was added to the Schlenk tube and the reaction stirred under $\mathrm{N}_{2}$ at $40^{\circ} \mathrm{C}$ for $24 \mathrm{~h}$. After initiation, the polymerisation underwent propagation by cooling to $0{ }^{\circ} \mathrm{C}$ before $N$-ethyldiisopropylamine in anhydrous DMF $(12.5 \mathrm{mg}$ $\mathrm{mL}^{-1}, 2 \mathrm{~mL}$ ) was added. After stirring at $0{ }^{\circ} \mathrm{C}$ with a flow of $\mathrm{N}_{2}$ for six days, the polymerisation reaction was finished as FTIR spectroscopy determined that Sar-NCA was no longer present. The nanocomposite was collected by vacuum, washed three times in chloroform (centrifugation for 30 minutes each time at $4500 \mathrm{rpm}$ ) and dried in a vacuum oven at $45{ }^{\circ} \mathrm{C}$.

\subsection{Synthesis of nanocomposite CND/CaNP-HUPA-PSar 17 and CND/CaNP-HUPA-PSar ${ }_{23}$ : the grafting-to approach}

Into a sample vial, the HUPA-PSar polymer (see ESI $\dagger$ ) $(150 \mathrm{mg}$ ) and CND/CaNPs (50 mg) were weighed and suspended in DI water $(10 \mathrm{~mL})$ with the aid of an ultrasonic bath for $1 \mathrm{~h}$. The nanocomposites were collected by centrifugation $(4500 \mathrm{rpm}$, $30 \mathrm{~min}$ ), washed with DI water three times, washed with ethanol three times, washed with chloroform three times, and dried in a vacuum oven at $45^{\circ} \mathrm{C}$.

\subsection{Dox release studies}

Firstly, Dox $\cdot \mathrm{HCl}$ ( $3 \mathrm{mg}, 5.2 \mu \mathrm{mol}$ ) was converted to its free base by dissolving in chloroform $(3 \mathrm{~mL})$ and adding triethylamine $(20 \mu \mathrm{L}, 14.5 \mathrm{mg}, 143 \mu \mathrm{mol})$ by micropipette on stirring. After $4 \mathrm{~h}$ of stirring at room temperature in the dark in a closed vial, a stock solution of Dox free base in chloroform was prepared.

To load the nanocomposites (3 mg) with Dox, they were added to DMF (1 mL). Phosphate buffered saline (PBS) solution at $\mathrm{pH} 7.4(10 \mathrm{~mL})$ was stirred vigorously whilst the nanocomposite in DMF solution was added dropwise simultaneously with the Dox free base in chloroform solution. The mixture was stirred vigorously overnight in the dark for the chloroform to evaporate, and the Dox-loaded nanocomposites transferred to dialysis tubing (2000 Da MWCO) and dialysed in the dark against PBS solution at $\mathrm{pH} 7.4$ at $37{ }^{\circ} \mathrm{C}$ until the excess Dox was removed. The concentration of Dox in the solution outside the dialysis tubing was quantified by HPLC against a calibration curve of known concentrations (Fig. S19†). Dox loading efficiency was calculated from eqn (1).

After dialysis, the solutions inside the dialysis tubing were split in two, with half dialysed against fresh PBS buffer solution at $\mathrm{pH}$ 7.4, and the other half dialysed against acetate buffer solution at $\mathrm{pH} 5$, both at $37^{\circ} \mathrm{C}$ in the dark. The buffer solution outside the dialysis tubing was taken at various time intervals, analysed by HPLC for Dox release quantification, and replaced with fresh buffer solution.

$$
\begin{aligned}
& \text { Dox loading of efficiency }=100 \% \\
& \times \frac{\text { mass of drug in nanocomposite }}{\text { mass of drug in feed }}
\end{aligned}
$$

\section{Results and discussion}

\subsection{CND/CaNP synthesis and functionalisation}

CND-loaded CaNPs (CND/CaNPs) were produced using a carbonation method to near-complete yields. ${ }^{44}$ Raman microscopy and pXRD confirmed that the CND/CaNPs produced were calcite (Fig. S1-S4†) with average diameters of $54 \pm$ $14 \mathrm{~nm}$, as determined by SEM analysis (Fig. S1a, $\dagger$ averaged over 126 particles), which was in suitable agreement with the value obtained from the Scherrer treatment of the pXRD pattern $(62 \pm 8 \mathrm{~nm}) .{ }^{45}$ The CND/CaNPs displayed bright blue fluorescence under UV radiation and a green afterglow visible for $\sim 1 \mathrm{~s}(\tau=\sim 0.25 \mathrm{~s})$ after the excitation source was removed (Fig. S1b $\dagger$ ).

ABPA and HUPA were used to prepare CND/CaNP surfaces for modification by NCA ROP. ${ }^{46}$ Both molecules feature phosphonic acid groups which interact with CaNP, while the hydroxyl or amine groups acts as the initiator for subsequent ROP (Scheme 1). FTIR analysis confirmed successful functionalisation of CND/CaNPs; the distinctive bands of calcite were found at $1397 \mathrm{~cm}^{-1}, 871 \mathrm{~cm}^{-1}$ and $713 \mathrm{~cm}^{-1}$, in the spectra of ABPA- and HUPA-functionalised CND/CaNPs (Fig. S4 ${ }^{-}$). Additionally, HUPA-functionalised CND/CaNPs possess $\mathrm{C}-\mathrm{H}$ stretching peaks at $2924 \mathrm{~cm}^{-1}$ and $2850 \mathrm{~cm}^{-1}$ corresponding with the eleven $\mathrm{CH}_{2}$ groups of HUPA. Peaks corresponding to $\mathrm{CH}_{2}$ groups could also be found in the spectrum of ABPA-functionalised CND/CaNPs, in addition to those corresponding to $\mathrm{P}-\mathrm{O}\left(1020 \mathrm{~cm}^{-1}\right)$ and $\mathrm{P}-\mathrm{O}-\mathrm{M}$ bonds $\left(1210 \mathrm{~cm}^{-1}\right)$. SEM analysis of the surface-functionalised CND/CaNPs showed that their morphology and dimensions are not visually altered by surface-functionalisation (Fig. S5†).

\subsection{CND/CaNP-poly(amino acid) nanocomposite synthesis}

Initially, amphiphilic block copolymers consisting of hydrophobic PPhe or PBLG and hydrophilic PSar (CND/ CaNP-ABPA-PPhe ${ }_{4}-b$-PSar ${ }_{16}$ and CND/CaNP-ABPA-PBLG 18 - $b$ PSar $_{16}$ ) were synthesised via simple, catalyst-free, sequential NCA ROP from ABPA-functionalised CND/CaNPs ('graftingfrom' Scheme 1, left).

PSar was also grafted from HUPA-functionalised CND/ CaNPs (CND/CaNP-HUPA-PSar ${ }_{82}$ ) to yield an amphiphilic block copolymer in which the alkane chain of HUPA acted as the hydrophobic component. NCA ROP proceeded from the hydroxyl group of HUPA, producing an ester link within the block copolymer chain (Scheme 1, right) that may be exploited for acid-mediated polymer cleavage and consequent guest molecule release. The nanocomposites produced by the grafting of NCA monomers from CND/CaNPs are summarised in 


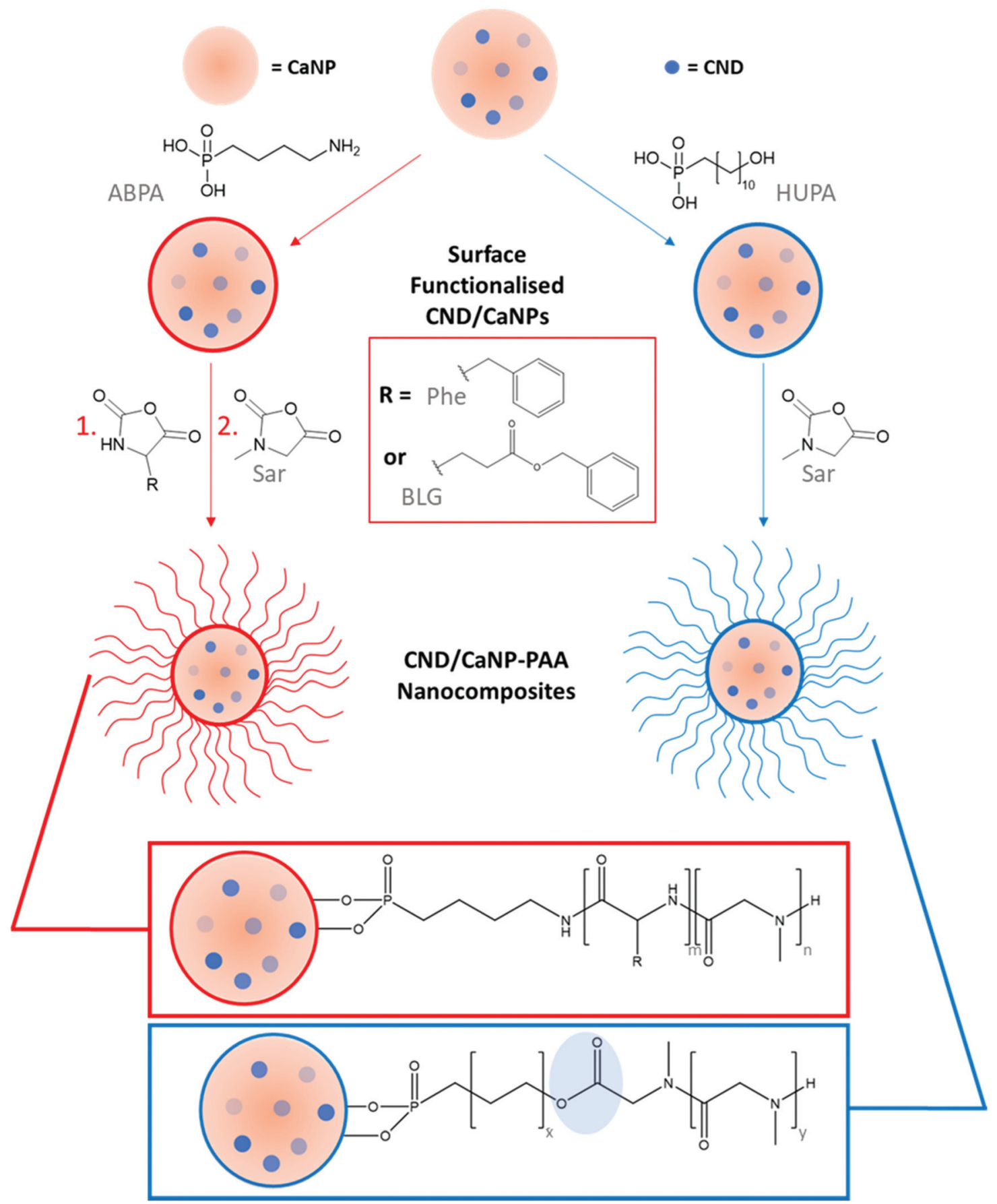

Scheme 1 (a) The creation of amino and hydroxyl-functionalised CaNPs that contain CNDs, followed by ROP to yield the nanocomposites CND/ CaNP-APBA-PPhe- $b$-PSar or CND/CaNP-APBA-PBLG-b-PSar (left) and CND/CaNP-HUPA-PSar (right). The ester link that renders CND/ CaNP-HUPA-PSar 82 susceptible to acid-catalysed hydrolysis is highlighted.

Table 1, entries 1-3. Comparing the integrals of the protons corresponding to the initiator (ABPA or HUPA) with the protons corresponding to the polymer allowed for quantification of polymer equivalents, and hence average polymer molecular weight values to be determined (Table $\mathrm{S} 1 \dagger$ ).

${ }^{1} \mathrm{H}$ NMR spectra were obtained to characterise polymeric surface coatings in comparison with spectra from similar
Table 1 Summary of nanocomposite materials synthesised

\begin{tabular}{lll}
\hline & Synthesis method & Material \\
\hline 1 & Grafting-from & CND/CaNP-ABPA-PPhe ${ }_{4}-b$-PSar \\
2 & Grafting-from & CND/CaNP-ABPA-PBLG \\
18 & $-b-$ PSar $_{16}$ \\
3 & Grafting-from & CND/CaNP-HUPA-PSar \\
4 & Non-covalent grafting-to & CND/CaNP-HUPA-PSar \\
4 & Non-covalent grafting-to & CND/CaNP-HUPA-PSar \\
5 & N &
\end{tabular}


unbound polymers, in terms of structure and monomer repeat units, formed in the absence of CND/CaNPs. Prior to all analyses, the CND/CaNP-polymer nanocomposites were suspended and extensively washed in chloroform, and then collected as a pellet by centrifugation. The supernatants were combined, dried, and the composition of the supernatants and pellets were analysed by ${ }^{1} \mathrm{H}$ NMR spectroscopy to establish their content. Successful polymer grafting is indicated by the presence of both polymer and CND/CaNPs in the pellet, whereas unbound polymer is present in the supernatant. This ensures that all peaks assigned to the polymer arise from CND/CaNP-conjugated polymer, as opposed to unbound polymer. ${ }^{1} \mathrm{H}$ NMR spectra of both bound $\mathrm{CND} /$ CaNP-ABPA-PPhe ${ }_{4}-b$ - PSar $_{16}$ and an unbound polymer analogue ABPA-PPhe ${ }_{8}-b$-PSar ${ }_{105}$ featured a peak between 7.34 and $7.12 \mathrm{ppm}$ which was assigned to the aromatic groups of the PPhe block (Fig. 1a and b). The peak between 4.50 and $3.92 \mathrm{ppm}$ corresponds to the proton of the amide group present in both polymer blocks, and peaks between 2.90 and $2.73 \mathrm{ppm}$ correspond to the protons of the methyl group bonded to the tertiary amine of each PSar repeat unit. The ${ }^{1} \mathrm{H}$ NMR spectra of CND/CaNP-ABPA-PBLG $18-b$-PSar 16 and the unbound polymer analogue ABPA-PBLG $22-b$ - PSar $_{63}$ both contained an aromatic peak between 7.37 and 7.20 ppm corresponding to the aromatic protons of the PBLG block (Fig. 1c and d). The peak between 5.12 and 5.00 ppm corresponds to the non-aromatic protons of the benzyl protecting group of each BLG repeat unit, and peaks between 2.95 and $2.71 \mathrm{ppm}$ correspond to the methyl group of each Sar repeat unit. This analysis, following extensive material washing, confirms that

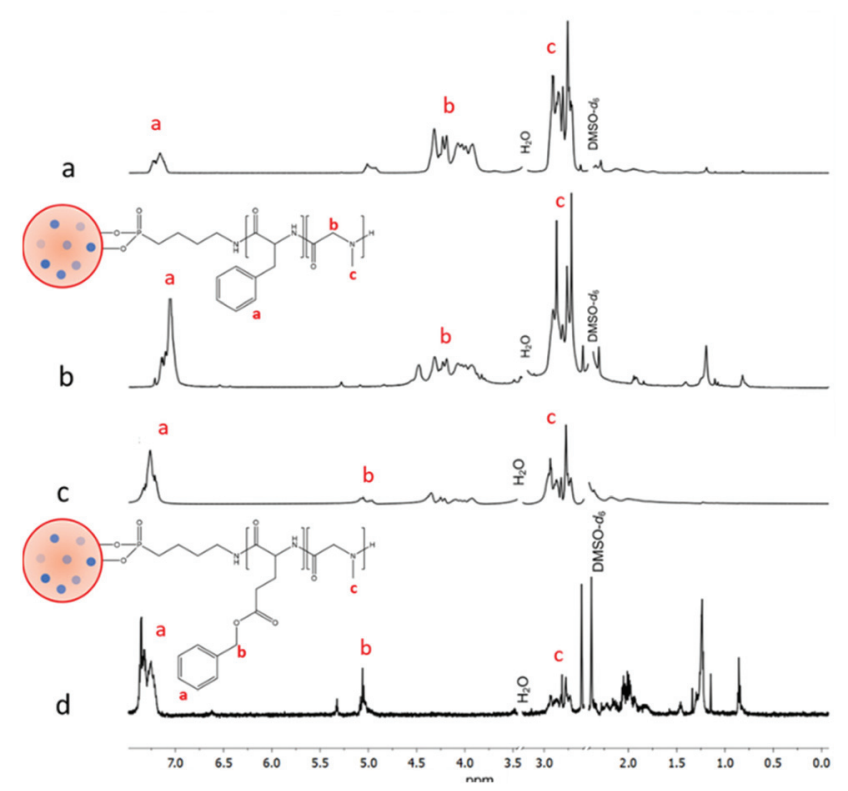

Fig. 1 (a) ${ }^{1} \mathrm{H}$ NMR spectra of ABPA-PPhe ${ }_{8}-b-$ PSar $_{105}(500 \mathrm{MHz}$, DMSO$\left.d_{6}\right)$, (b) CND/CaNP-ABPA-PPhe $4-b-$ PSar $_{16}\left(600 \mathrm{MHz}, \mathrm{DMSO}-d_{6}\right)$, (c) ABPA-PBLG $22-b-$ PSar $_{63}\left(500 \mathrm{MHz}\right.$, DMSO- $\left.d_{6}\right)$, and (d) CND/ CaNP-ABPA-PBLG $18-b-$ PSar $_{16}\left(600 \mathrm{MHz}\right.$, DMSO- $\left.d_{6}\right)$. Key peaks corresponding to poly(amino acid) protons are denoted. both PPhe $_{4}-b$-PSar 16 and $\mathrm{PBLG}_{18}-b-\mathrm{PSar}_{16}$ are bound to the CND/CaNPs.

PSar grafting from HUPA-modified CND/CaNPs was also confirmed by ${ }^{1} \mathrm{H}$ NMR spectroscopy, with unbound HUPA-PSar $_{95}$ used as a reference (Fig. 2). Both spectra contained a peak between $4.33 \mathrm{ppm}$ and $3.92 \mathrm{ppm}$ corresponding to the $\mathrm{CH}$ group of the Sar repeat unit, a peak between 2.92 and $2.73 \mathrm{ppm}$ corresponding to the methyl group of the Sar repeat unit, and peaks between 1.24 and 1.07 ppm corresponding to the $\mathrm{CH}_{2}$ groups of HUPA. ${ }^{13} \mathrm{C}$ NMR spectroscopy confirmed successful nanocomposite synthesis (Fig. S6-S8†). ${ }^{13} \mathrm{C}$ NMR peaks corresponding to the polymeric component are present in all spectra. In contrast, the peak at $168.21 \mathrm{ppm}$, corresponding to calcite, ${ }^{47}$ appeared only in the spectra obtained from CND/CaNP-polymer nanocomposite.

Raman spectra obtained from bare CND/CaNPs featured characteristic calcite peaks at $1086 \mathrm{~cm}^{-1}$ and $711 \mathrm{~cm}^{-1}$ (Fig. S2†). Raman spectra obtained from CND/CaNP-polymer nanocomposites also contained these peaks, and a characteristic aromatic peak at $1002 \mathrm{~cm}^{-1}$ which suggests that polymer grafting caused no structural changes to the calcite core (Fig. S9†). The dried supernatant from the washing process revealed a Raman spectra comparable to those obtained from free polymer and featured no peaks corresponding to calcite, further confirming that chloroform washing successfully removed unbound polymer from polymer-grafted CND/CaNPs (Fig. S10†).

FTIR spectroscopy was used for additional characterisation of the bare CND/CaNP nanoparticles and CND/CaNP-polymer nanocomposites (Fig. 3). The spectra of all materials revealed the characteristic calcite peaks at $1397 \mathrm{~cm}^{-1}, 871 \mathrm{~cm}^{-1}$ and $713 \mathrm{~cm}^{-1}$, and an amide peak in the spectra of polymergrafted nanoparticles. Once more, thorough washing with chloroform prior to FTIR, Raman and ${ }^{13} \mathrm{C}$ NMR analysis confirmed that the polymer remains grafted to the CND/CaNPs.

The capability of poly(amino acids) featuring a terminal phosphonate group to graft to CND/CaNPs via ionic interactions was then investigated ('non-covalent grafting-to' approach). Two phosphonate-bearing PSar polymers were synthesised via NCA ROP initiated from the hydroxyl group of

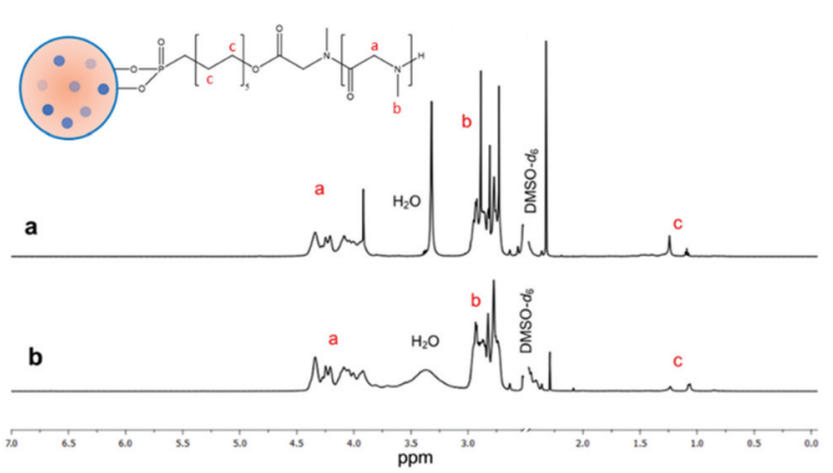

Fig. 2 (a) ${ }^{1} \mathrm{H} \quad \mathrm{NMR}$ spectra of CND/CaNP-HUPA-PSar 82 and (b) HUPA-PSar 95 (500 MHz, DMSO- $d_{6}, \delta$ ppm). 


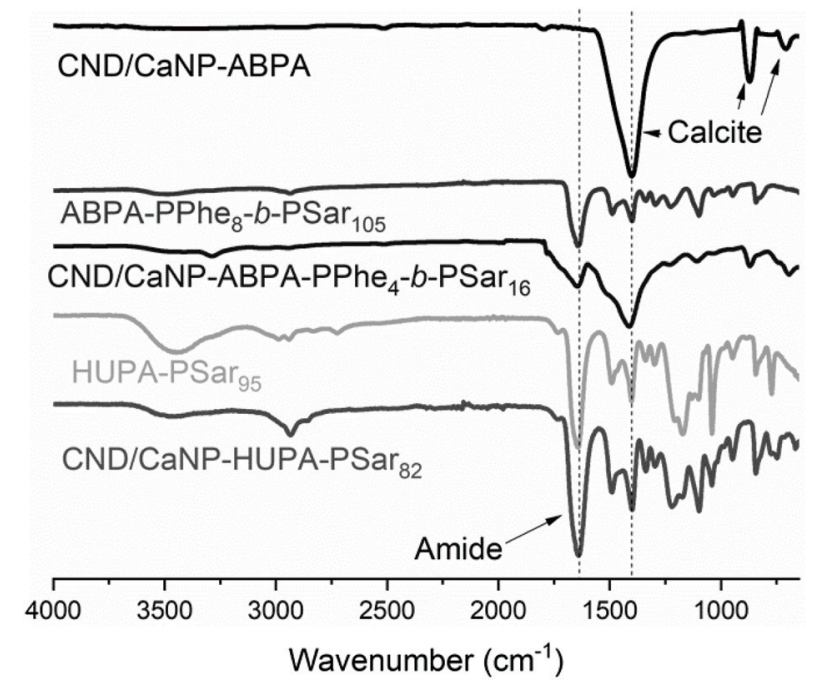

Fig. 3 FTIR spectra of ABPA-functionalised CND/CaNPs, ABPA-PPhe ${ }_{8}$ $b$-PSar 105 free polymer, CND/CaNP-ABPA-PPhe ${ }_{4}-b-$ PSar $_{16}$, HUPA-PSar ${ }_{95}$ free polymer and CND/CaNP-HUPA-PSar 82.

HUPA: HUPA-PSar ${ }_{17}$ and HUPA-PSar ${ }_{23}$ (Table 1). ${ }^{1} \mathrm{H}$ NMR (Fig. S11†), ${ }^{13} \mathrm{C}$ NMR (Fig. S12†) and FTIR (Fig. S13†) spectroscopies confirmed successful polymer synthesis. The noncovalent grafting-to approach to nanocomposite creation enables straightforward polymer analysis by ${ }^{1} \mathrm{H}$ NMR spectroscopy in solution owing to the solubility of the polymers when not grafted to an inorganic nanoparticle. The polymers were then introduced to CND/CaNPs as an aqueous solution and suspended using an ultrasonic bath. After $1 \mathrm{~h}$, the nanocomposite samples were processed and analysed as described above. FTIR spectra of both chloroform-washed nanocomposites featured characteristic broad peaks attributed to calcite $\left(1397 \mathrm{~cm}^{-1}, 871 \mathrm{~cm}^{-1}\right.$ and $\left.713 \mathrm{~cm}^{-1}\right)$ and the amide from the polymer $\left(1634 \mathrm{~cm}^{-1}\right.$, Fig. S14†) confirming nanocomposite self-assembly. Meanwhile, FTIR analysis indicated that unbound polymer was washed away and retained in the chloroform supernatant (Fig. S15†).

The extent of polymer grafting to CND/CaNPs was explored using thermogravimetric analysis (TGA, Table 2 and Fig. S16†). The thermal decomposition of CND/CaNPs occurred with a single weight loss step between $600{ }^{\circ} \mathrm{C}$ and $700{ }^{\circ} \mathrm{C}$, leaving a

Table 2 Summary of TGA data. The furnace temperatures recorded at $95 \%, 90 \%$ and $60 \%$ weight loss, and the char yields at $800{ }^{\circ} \mathrm{C}$

\begin{tabular}{|c|c|c|c|c|}
\hline \multirow[b]{2}{*}{ Sample } & \multicolumn{3}{|c|}{$\begin{array}{l}\text { Temperature at } \\
\text { wt } \%\left({ }^{\circ} \mathrm{C}\right)\end{array}$} & \multirow{2}{*}{$\begin{array}{l}\text { Char yield at } \\
\left.800{ }^{\circ} \mathrm{C} \text { (wt } \%\right)\end{array}$} \\
\hline & $95 \%$ & $90 \%$ & $60 \%$ & \\
\hline CND/CaNP & 634 & 662 & 723 & 58 \\
\hline CND/CaNP-ABPA-PPhe ${ }_{4}-b-$ PSar $_{16}$ & 246 & 281 & 410 & 31 \\
\hline ABPA-PPhe $_{8}-b$-PSar $_{105}$ & 234 & 246 & 271 & 2 \\
\hline CND/CaNP-HUPA-PSar 23 & 219 & 300 & 659 & 46 \\
\hline HUPA-PSar $_{95}$ & 122 & 155 & 238 & 3 \\
\hline
\end{tabular}

yield of $58 \%$ at $800{ }^{\circ} \mathrm{C}$ which corresponds to the loss of one formula unit $\mathrm{CO}_{2}$ per formula unit $\mathrm{CaCO}_{3}$. In contrast, pure polymers ABPA-PPhe ${ }_{8}-b$-PSar ${ }_{105}$ and HUPA-PSar ${ }_{95}$ showed a dominant weight loss step which started between $250^{\circ} \mathrm{C}$ and $350{ }^{\circ} \mathrm{C}$, and was completed at $\sim 550{ }^{\circ} \mathrm{C}$. Negligible char remained at $800{ }^{\circ} \mathrm{C}$. Therefore, it was possible to determine the extent of polymer grafting by comparing the weight loss at lower (attributed to polymer decomposition) and higher (attributed to CND/CaNPs decomposition) temperatures.

TGA data showed that the total organic material mass of CND/CaNP-ABPA-PPhe ${ }_{4}-b$-PSar ${ }_{16}$ (grafting-from approach) and CND/CaNP-HUPA-PSar ${ }_{23}$ (non-covalent grafting-to approach) was $27 \%$ and $12 \%$, respectively, suggesting that the graftingfrom approach enabled more efficient polymer attachment to CND/CaNPs surfaces. Consequently, nanocomposites formed by the grafting-from approach were advanced to controlled therapeutic release studies.

\subsection{Nanocomposites as drug release vehicles}

Drug delivery systems intended for intravenous injection must form a stable dispersion for extended shelf-life prior to administration, and disperse in aqueous solution, such as the bloodstream, post-administration. The effect of polymer grafting on enhancing the dispersibility of CND/CaNPs in water was then tested by time-resolved turbidimetry studies. This analysis involved monitoring a peak at $650 \mathrm{~nm}$ in the UV-Vis spectrum that was a consequence of turbidity caused by the nanoparticle dispersion. Upon nanoparticle aggregation, the aggregates sink to the bottom of the cuvette, resulting in a clear solution and a reduction in peak intensity. Only $11 \% \mathrm{CND} / \mathrm{CaNPs}$ remained homogenously dispersed in water after $4 \mathrm{~h}$, whereas $34 \%$ of $\mathrm{CND} / \mathrm{CaNP}-\mathrm{ABPA}-\mathrm{PPhe}_{4}-b-\mathrm{PSar}_{16}$ and $82 \%$ of $\mathrm{CND} /$ CaNP-HUPA-PSar ${ }_{23}$ remained homogeneously dispersed in water after $4 \mathrm{~h}$ (Fig. S17†). The increased stability of the suspension was attributed to the introduction of a hydrophilic PSar shell to the CND/CaNP surface. The difference in suspension stability enhancement between the two classes of nanocomposites may be linked to the differences in PSar chain length, the bulky hydrophobic phenyl groups in the PPhe chain length, and the extent of polymer grafting. Nevertheless, polymer grafting enhanced the stability of $\mathrm{CND} / \mathrm{CaNP}$ dispersions in water.

Owing to the nanocomposites improved suspension stability over CND/CaNPs, CND/CaNP-ABPA-PPhe ${ }_{4}-b$-PSar ${ }_{16}$ and CND/CaNP-HUPA-PSar ${ }_{23}$ were assessed for their ability to both retain and selectively release the chemotherapeutic Dox. The nanocomposites were suspended in $\mathrm{pH}$ 7.4 PBS buffer solution and loaded with Dox using the 'dropping-in' method, ${ }^{48}$ to $82 \%$ and $72 \%$ efficiency, respectively (eqn (1)). Dox-loaded nanocomposite suspensions were then dialysed against $\mathrm{pH}$ 7.4 PBS solution while the Dox concentration in the surrounding dialysis medium was assayed over time by HPLC (Fig. 4). Negligible Dox $(0.0-0.1 \%)$ was released after $168 \mathrm{~h}$ from both nanocomposites, suggesting excellent retention of Dox under physiological $\mathrm{pH}$ conditions. It was also inferred that both the polymer shell remained intact and attached to the CaNP core, 

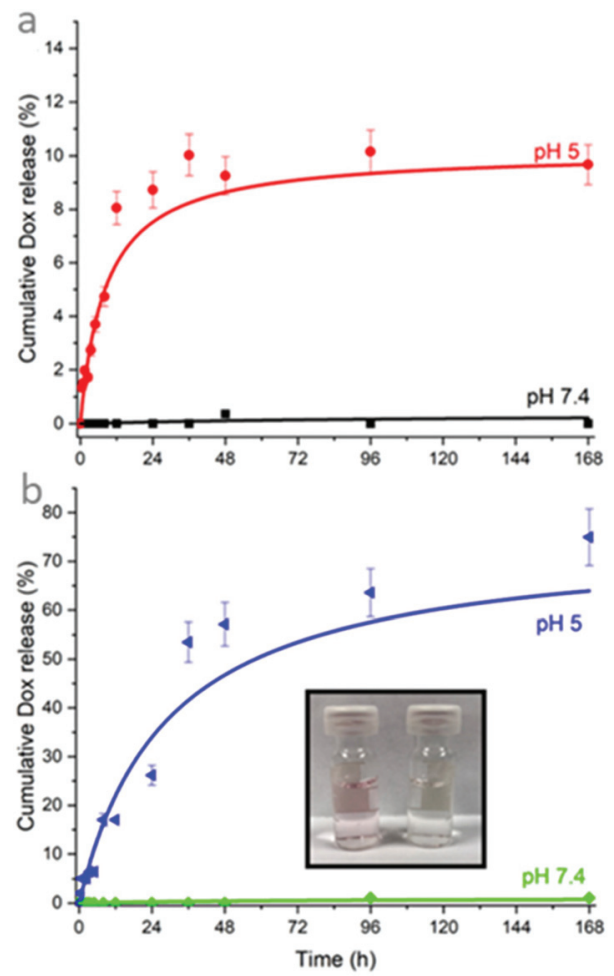

Fig. 4 (a) Cumulative amount of Dox released over time from CND/ CaNP-ABPA-PPhe ${ }_{4}-b-$ PSar $_{16}$ in PBS buffer solution at $\mathrm{pH} 7.4$ (घ) and acetate buffer solution at $\mathrm{pH} 5$ (o). (b) Cumulative amount of Dox released over time from CND/CaNP-HUPA-PSar 23 in PBS buffer solution at $\mathrm{pH} 7.4(\diamond)$ and acetate buffer solution at pH $5(\measuredangle)$. Inset: Buffer solutions outside the dialysis tubing of CND/CaNP-HUPA-PSar 23 at $\mathrm{pH} 5$ (left) and 7.4 (right) after $168 \mathrm{~h}$.

preventing Dox passage to the external solution, and that the CaNP core was protected from dissolution into the surrounding medium.

To test the capability for the nanocomposites to release Dox as triggered by a $\mathrm{pH}$ change, Dox-loaded nanocomposite suspensions were also dialysed against a pH 5 acetate buffer. Only moderate Dox release from CND/CaNP-ABPA-PPhe ${ }_{4}-b-$ PSar $_{16}$ was anticipated due to the inability of acid to hydrolyse the polymer at such pH; Dox release may instead be driven by its protonation and enhanced solubility in the acidic environment, enabling some escape from the nanoparticle. In addition, some degradation of the CaNP core is anticipated due to the considerably higher solubility of $\mathrm{CaCO}_{3}$ at an acidic $\mathrm{pH}$, but the remaining surface-bound phosphonate-containing molecule is likely to hinder the dissolution of the CaNP core. Accordingly, 9.6\% of the loaded Dox was released (Fig. 4a). In contrast, Dox release from CND/CaNP-HUPA-PSar ${ }_{23}$ was hypothesised to be significant due to the presence of the acidcleavable ester link between the HUPA and PSar blocks within the polymer shell. Studies revealed that $74.9 \%$ of the loaded Dox was released when the nanocomposites were maintained within pH 5 acetate buffer solution. The pH-triggered release of Dox from both nanocomposites was mostly completed within the first $24 \mathrm{~h}$ of the experiment, indicating a restricted 'burst release' (Fig. 4b). Furthermore, the release of Dox was also clearly demonstrated by the colourless-to-pink colour change observed in the pH 5 acetate buffer dialysis medium, attributed to the increasing Dox concentration. In contrast, no colour change was observed when $\mathrm{pH}$ 7.4 PBS solution was used as the dialysis medium (Fig. 4b, inset).

Such selectivity in drug release is extremely advantageous to drug delivery vehicles that are intended to release guest molecules only at the target site. ${ }^{49}$ However, the ability to trace the activity of a drug delivery vehicle in real time is also desirable for ensuring the therapeutic reaches the target site. Here, the nanocomposites feature a CND/CaNP core which exhibits a green afterglow after UV stimulation when intact, but loses this ability when dissolved and the CNDs are instead in aqueous solution. Therefore, introducing the nanocomposites to an acidic environment would not only trigger Dox release, but it may also provide a mechanism for nanocomposite tracing if the loss of afterglow behaviour was traced by identifying areas where the afterglow disappears over time as the nanocomposite degrades. Stroboscopy studies showed that the nanocomposite CND/CaNP-ABPA-PPhe 4 - $b$ - PSar $_{16}$ retained the afterglow behaviour of the core when suspended in $\mathrm{pH}$ 7.4 PBS buffer solution, showing that it was unaffected by the polymer shell (Fig. 5a). Afterglow behaviour was also retained after the nanocomposites were recovered from solution and dried

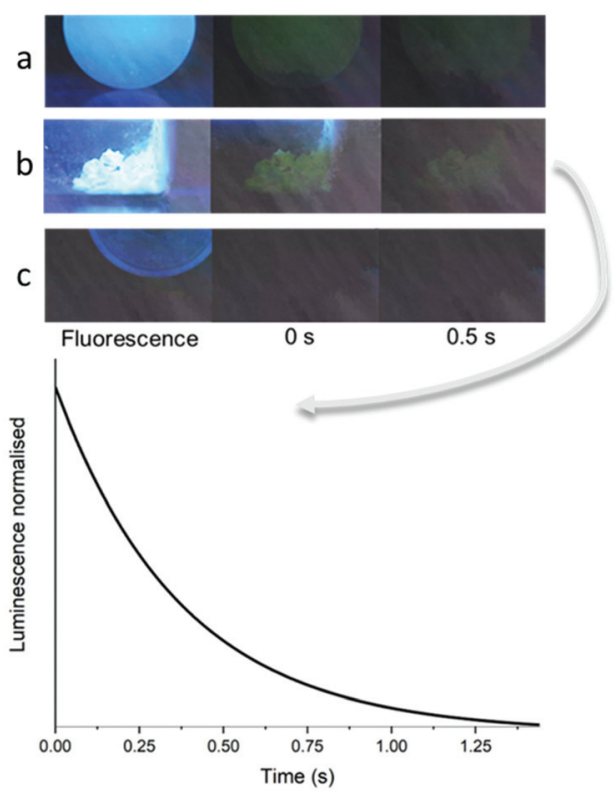

Fig. 5 Images determining the presence of afterglow of (a) CND/ CaNP-ABPA-PPhe ${ }_{4}-b-$ PSar $_{16}$ in pH 7.4 PBS buffer solution, (b) CND/ CaNP-ABPA-PPhe ${ }_{4}-b-\mathrm{PSar}_{16}$ following removal from solution and drying, (c) CND/CaNP-ABPA-PPhe ${ }_{4}-b$-PSar ${ }_{16}$ suspended in $\mathrm{pH} 5$ acetate buffer solution. The normalised afterglow lifetime of CND/ CaNP-ABPA-PPhe $4-b-$ PSar $_{16}$ following removal from solution is presented below the microscopy images. Afterglow was triggered by irradiating the samples with UV light at $365 \mathrm{~nm}$ for a few seconds beforehand. 
(Fig. 5b). To determine the response of afterglow behaviour to acidic solution, the same nanocomposites were suspended in pH 5 acetate buffer solution, stirred for $24 \mathrm{~h}$ and then dried. No afterglow was observed for any nanocomposites tested, which was ascribed to the degradation of the CaNP core (Fig. 5c). We propose that the combination of $\mathrm{pH}$-responsive nature of afterglow and Dox release offers a system for tracked and controlled payload release. However, it is remarked that CNDs with more suitable photophysical characteristics are required to use our material design concept for tracing studies in vivo. This includes excitation with red light and emission in red/NIR to enable efficient transmission of light through living tissue, and an optimised afterglow quantum yield to account for dilution. Research into the production and optimisation of CNDs for this purpose is currently ongoing.

\section{Conclusion}

CND/CaNPs were functionalised with both ABPA and HUPA to enable amphiphilic block copolymers to be grafted from the nanoparticles by NCA ROP. Additionally, amphiphilic HUPA-PSar block copolymers were synthesised, and grafted to $\mathrm{CND} / \mathrm{CaNPs}$ by the phosphonate functional group forming $\mathrm{P}-$ O-M bonds with CaNPs. These synergistic CND/CaNP-polymer nanocomposites had good compatibility with aqueous media whilst retaining fluorescence and afterglow. The nanocomposites were loaded with Dox at high efficiency and Dox release studies revealed that an acid environment was able to promote Dox release from the nanocomposites, particularly when an ester link was incorporated within the polymer chain. The selectivity of the nanocomposites to release Dox to an acidic environment, but retain Dox when stored in a nonacidic environment, was significant, and is a key requirement of macromolecular vehicles for controlled drug release use. The nanocomposites demonstrated afterglow in $\mathrm{pH} 7.4$ solution up to approximately $1 \mathrm{~s}$, but this was lost when the nanocomposites were stored in acidic $\mathrm{pH}$. This could be optimised further as a tracing mechanism by watching for the loss of afterglow in target cells.

\section{Author contributions}

J. V. R.: project coordination, overall material synthesis and analysis, producer of the first draft of the manuscript. P. A. W.: assistance with nanocomposite synthesis and analysis. H. Y.: assistance with monomer and polymer synthesis and analysis. M. J. H.: solution-state NMR spectroscopy analysis and assistance in data analysis. D. L. B.: solid-state NMR spectroscopy analysis and assistance in data analysis. A. K.: Raman spectroscopy, TGA, and SEM analysis. D. C. G.: conceptualisation, project supervision, writing - review and editing. P. D. T.: conceptualisation, project supervision, writing - review and editing.

\section{Conflicts of interest}

There are no conflicts of interest to declare.

\section{Acknowledgements}

The authors would like to thank the University of Leeds for financial assistance via the Gunnell and Matthews Scholarship, the EPSRC for providing funding to David C. Green via the research grant EP/P005233/1, and Martin Huscroft and Jeanine Williams for HPLC analysis.

\section{Notes and references}

1 A. Roy, P. P. Maity, S. Dhara and S. Pal, J. Appl. Polym. Sci., 2018, 135, 45939.

2 N. Permyakova, T. Zheltonozhskaya, M. Ignatovskaya, V. Maksin, O. Iakubchak and D. Klymchuk, Colloid Polym. Sci., 2018, 296, 295-307.

3 W. Ke, W. Yin, Z. Zha, J. F. Mukerabigwi, W. Chen, Y. Wang, C. He and Z. Ge, Biomaterials, 2018, 154, 261-274.

4 M. Appold, C. Mari, C. Lederle, J. Elbert, C. Schmidt, I. Ott, B. Stühn, G. Gasser and M. Gallei, Polym. Chem., 2017, 8, 890-900.

5 C. Shi, C. Thum, Q. Zhang, W. Tu, B. Pelaz, W. J. Parak, Y. Zhang and M. Schneider, J. Controlled Release, 2016, 237, 50-606.

6 H. Yu, J. V. Rowley, D. C. Green and P. D. Thornton, Mater. Adv., 2020, 1, 1293-1300.

7 P. D. Thornton and A. Heise, Chem. Commun., 2011, 47, 3108-3110.

8 Z. Gao, B. Gotland, G. Tronci and P. D. Thornton, J. Mater. Chem. B, 2019, 7, 7494-7501.

9 B. S. McAvan, M. Khuphe and P. D. Thornton, Eur. Polym. J., 2017, 87, 468-477.

10 M. Thompson and C. Scholz, Nanomaterials, 2021, 11, 1119.

11 M. Khuphe, C. S. Mahon and P. D. Thornton, Biomater. Sci., 2016, 4, 1792.

12 J. Huang and A. Heise, Chem. Soc. Rev., 2013, 42, 73737390 .

13 N. Hadjichristidis, H. Iatrou, M. Pitsikalis and G. Sakellariou, Chem. Rev., 2009, 109, 5528-5578.

14 R. Baumgartner, H. Fu, Z. Song, Y. Lin and J. Cheng, Nat. Chem., 2017, 9, 614.

15 M. Khuphe, A. Kazlauciunas, M. Huscroft and P. D. Thornton, Chem. Commun., 2015, 51, 1520-1523.

16 S. G. Roy, U. Haldar and P. De, ACS Appl. Mater. Interfaces, 2014, 6, 4233-4241.

17 S. Zheng, Y. H. Guan, H. C. Yu, G. Huang and C. J. Zheng, New J. Chem., 2019, 43, 9989-10002.

18 T. Borase, M. Iacono, S. I. Ali, P. D. Thornton and A. Heise, Polym. Chem., 2012, 3, 1267-1275. 
19 M. Q. Gong, J. L. Wu, B. Chen, R. X. Zhuo and S. X. Cheng, Langmuir, 2015, 31, 5115-5122.

20 B. Chang, X. Sha, J. Guo, Y. Jiao, C. Wang and W. Yang, J. Mater. Chem., 2011, 21, 9239-9247.

21 S. Senapati, A. K. Mahanta, S. Kumar and P. Maiti, Signal Transduct. Target. Ther., 2018, 3, 7.

22 A. M. Derfus, W. C. W. Chan and S. N. Bhatia, Nano Lett., 2004, 4, 11-18.

23 H. T. Li, Z. H. Kang, Y. Liu and S.-T. Lee, J. Mater. Chem., 2012, 22, 24230-24253.

24 Y. X. Fang, S. J. Guo, D. Li, C. Z. Zhu, W. Ren, S. J. Dong and E. K. Wang, ACS Nano, 2012, 6, 400-409.

25 Q. Zeng, D. Shao, X. He, Z. Ren, W. Ji, C. Shan, S. Qu, J. Li, L. Chen and Q. Li, J. Mater. Chem. B, 2016, 30, 5119-5126.

26 F. Wang, Y.-h. Chen, C.-y. Liu and D.-g. Ma, Chem. Commun., 2011, 47, 3502-3504.

27 L. Cao, S. Sahu, P. Anilkumar, C. E. Bunker, J. Xu, K. A. S. Fernando, P. Wang, E. A. Guliants, K. N. Tackett and Y. P. Sun, J. Am. Chem. Soc., 2011, 133, 4754-4757.

28 H. T. Li, X. D. He, Z. H. Kang, H. Huang, Y. Liu, J. L. Liu, S. Y. Lian, C. H. A. Tsang, X. B. Yang and S.-T. Lee, Angew. Chem., Int. Ed., 2010, 49, 4430-4434.

29 Y. Chen, J. He, C. Hu, H. Zhang, B. Lei and Y. Liu, J. Mater. Chem. C, 2017, 5, 6243-6250.

30 J. Tan, R. Zou, J. Zhang, W. Li, L. Zhang and D. Yue, Nanoscale, 2016, 8, 4742-4747.

31 D. C. Green, M. A. Holden, M. A. Levenstein, S. H. Zhang, B. R. G. Johnson, J. G. Pablo, A. Ward, S. W. Botchway and F. C. Meldrum, Nat. Commun., 2019, 10, 206.

32 Y. Fan, P. Wang, Y. Lu, R. Wang, L. Zhou, X. Zheng, X. Li, J. A. Piper and F. Zhang, Nat. Nanotechnol., 2018, 13, 941-946.

33 X. Zhu, X. Liu, H. Zhang, M. Zhao, P. Pei, Y. Chen, Y. Yang, L. Lu, P. Yu, C. Sun, J. Ming, I. M. Ábrahám, A. M. El-Toni, A. Khan and F. Zhang, Angew. Chem., Int. Ed., 2021, 60, 23545-23551.
34 W. Li, S. Wu, X. Xu, J. Zhuang, H. Zhang, X. Zhang, C. Hu, B. Lei, C. F. Kaminski and Y. Liu, Chem. Mater., 2019, 31, 9887-9894.

35 Z. Dong, L. Feng, W. Zhu, X. Sun, M. Gao, H. Zhao, Y. Chao and Z. Liu, Biomaterials, 2016, 110, 60-70.

36 D. H. Keum, J. H. Mun, B. W. Hwang, J. Kim, H. Kim, W. Jo, D. H. Ha, D. W. Cho, C. Kim and S. K. Hahn, Small, 2017, 13, 1602925.

37 S. Guo, M. Yang, M. Chen, J. Zhang, K. Liu, L. Ye and W. Gu, Dalton Trans., 2015, 44, 8232-8237.

38 M. A. Osman and U. W. Suter, Chem. Mater., 2002, 14, 4408-4415.

39 Z. Wang, J. Appl. Polym. Sci., 1996, 60, 2239-2243.

40 Z. Demjén, B. Pukánszky, E. Foldes and J. Nagy, J. Colloid Interface Sci., 1997, 190, 427-436.

41 Y. Mima, Y. Hashimoto, T. Shimizu, H. Kiwada and T. Ishida, Mol. Pharm., 2015, 12(7), 2429-2435.

42 Š. Gradišar, E. Žagar and D. Pahovnik, ACS Macro Lett., 2017, 6, 637-640.

$43 \mathrm{H}$. Yu, N. Ingram, J. V. Rowley, D. C. Green and P. D. Thornton, Chem. - Eur. J., 2020, 26, 1335213358.

44 B. Hari, X. Ding, Y. Guo, Y. Deng, C. Wang, M. Li and Z. Wang, Mater. Lett., 2006, 60, 1515-1518.

45 B. D. Cullity and S. R. Stock, Elements of X-ray diffraction, Prentice Hall, New Jersey, 2001.

46 W. El Malti, D. Laurencin, G. Guerrero, M. E. Smith and P. H. Mutin, J. Mater. Chem., 2012, 22, 1212-1218.

47 H. Nebel, M. Neumann, C. Mayer and M. Epple, Inorg. Chem., 2008, 47, 7874-7879.

48 D. J. Price, M. Khuphe, R. P. W. Davies, J. R. McLaughlan, N. Ingram and P. D. Thornton, Chem. Commun., 2017, 53, 8687-8690.

49 S. Mura, J. Nicolas and P. Couvreur, Nat. Mater., 2013, 12, 991-1003. 